\title{
Characterisation of extrasolar planetary transit candidates
}

\section{The companions to late M-type stars}

\author{
J. Gallardo ${ }^{1}$, S. Silva $^{1}$, S. Ramírez Alegría ${ }^{2,3}$, D. Minniti ${ }^{2,4}$, and P. Pietrukowicz ${ }^{2,5}$ \\ 1 Departamento de Astronomía, Universidad de Chile, Casilla 36-D, Santiago, Chile \\ e-mail: gallardo@das.uchile.cl \\ 2 Departamento de Astronomía y Astrofísica, P. Universidad Católica de Chile, Chile \\ Instituto Astrofísica de Canarias, 38200 La Laguna, Tenerife, Spain \\ 4 Vatican Observatory, 00120 Vatican City State, Italy \\ 5 Nicolaus Copernicus Astronomical Center, ul. Bartycka 18, 00-716 Warszawa, Poland
}

Received 30 November 2009 / Accepted 30 April 2010

\section{ABSTRACT}

\begin{abstract}
We present a second paper of a complete characterization of stars whose low-depth transits were discovered by the OGLE-III campaign to select the most promising candidates for spectroscopic confirmation. This paper follows a similar analysis to one previously performed in Gallardo et al. (2005, A\&A, 431, 707). We present new optical and near-IR photometry and derive physical parameters such as effective temperature $\left(T_{\text {eff }}\right)$, distance $(d)$, the stellar radii $\left(R_{*}\right)$, and the companion radii $\left(R_{\mathrm{c}}\right)$. We selected eight $\mathrm{M}(2800 \mathrm{~K}$ $\left.\leq T_{\text {eff }} \leq 3850 \mathrm{~K}\right)$ or $\mathrm{K}\left(3850 \mathrm{~K} \leq T_{\text {eff }} \leq 5150 \mathrm{~K}\right)$ stellar objects as potential candidates to host exoplanets, even though, considering the radii of their companions, only the stars OGLE-TR-61, OGLE-TR-74, OGLE-TR-123, and OGLE-TR-173 are the most promising M-type transit candidates to host planets. A confirmation of the planetary nature of any of these objects would be extremely valuable in providing a means of characterizing planets transiting M-type dwarfs with effective temperatures down to only $2900 \mathrm{~K}$.
\end{abstract}

Key words. planetary systems - stars: late-type - binaries: eclipsing

\section{Introduction}

The first transiting extrasolar planet was announced in 2000, when Charbonneau et al. (2000) and Henry et al. (2000) confirmed, by means of radial velocity measurements, the planetary nature of the transit light curve of HD 209458b. As of February 2010, there are approximately 69 extrasolar planets that have been found to transit their parent stars. Many of these planetary companions have been discovered by various photometric extra-solar planets surveys, such as OGLE (optical gravitational lensing experiment), STARE (STellar Astrophysics \& Research on Exoplanets), WASP (wide angle search for planets), and by the COROT space telescope (COnvection, ROtation \& planetary Transits, launched at the end of 2006), which claimed out the discovered of the first telluric planet (Léger et al. 2009). Despite the success of these projects, a major problem in identifying transiting planets has been that photometry alone does not allow us to unambiguously distinguish between extrasolar planets and other astrophysical low-luminosity objects such as very late M-type stars, brown dwarf, or grazing binaries, because all these objects have similar characteristics (e.g., Dreizler et al. 2002, 2007; Gallardo et al. 2005; Silva \& Cruz 2006). Transit surveys are able to monitor orders of magnitude more stars in a comparatively shorter observing time than radial velocity surveys, hence have the ability to discover many interesting exoplanet candidates, although non-planetary events must be filtered out. Once the classification has been performed, precise spectroscopic observations enable us to measure all the important parameters such as radii and masses.

Several studies have presented efficient ways of selecting the most promising planetary candidates from transit surveys. For instance, Dreizler et al. $(2002,2007)$ performed a selection using low dispersion spectroscopy, and Drake (2003) used the gravity darkening dependence of the light curve. Alonso et al. (2004) presented different configurations that can produce signals resembling those produced by transiting planets, and described various ways of several strategies to recognize these false alarms. They suggested that multicolor photometry is useful for identifying, for example, eclipsing binaries. Near-IR photometry may also be used to identify and remove giant stars and stars with an IR excess and larger companions identify by observing host-star ellipsoidal variability (e.g., Sirko \& Paczynski 2003). Gallardo et al. (2005) developed a method based on the well-calibrated surface brightness relation along with the correlation between mass and luminosity for main-sequence stars. This allows not only the classification of the most promising candidates, but also the measurement of the effective temperature and radius of the parent star.

As already mentioned, the OGLE photometric survey project (Udalski 2002, 2003, 2004) has been an efficient means of discovering transiting extrasolar planets, proving that the photometric detection of exoplanets can be successfull. During the years 2003 and 2005, the "planetary and low-luminosity companion" catalogues were published by the OGLE team. In these campaigns, several fields of the Galactic disk were monitored to search for low-depth transits, and 113 objects were discovered with transit depths shallower than 0.08 mag in the $I$ band.

In this work, which represents the continuation of the work published by Gallardo et al. (2005), we present new optical and near-IR photometry and measure the properties of 27 stars with observed transits (among the 113 just mentioned), constraining astrophysical parameters of both stellar and companion objects. 
We use the method developed by Gallardo et al. (2005) and focus our analysis on the G, K, and M-type parent stars. We select the most interesting stellar objects to host extrasolar planets from the depth in its transit light curve, and then derive stellar parameters such as effective temperature, stellar radius, and distance.

The paper is organized as follows. In Sect. 2, we present our observations, and we describe our data reduction in Sect. 3. In Sect. 4, we briefly discuss the method we used to determine both stellar and companion physical parameters, and the inferred properties are presented in Sect. 5. Our conclusions are given in Sect. 6.

\section{Observations}

In April and May 2005, we carried out several observations of OGLE extrasolar planetary transit candidates identified from its campaigns during the years 2003 and 2005. We observed every target for 2 or $3 \mathrm{~h}$ (acquiring about 100 images for a single star). A total of 14 stars were observed to show evidence of planetary transits in their light curves. We complemented these data with observations ( 5 images per star) of other OGLE stars to obtain near-IR photometry, and produced a near-IR photometric catalogue of the OGLE candidates. We used the SoFI IR camera at the ESO New Technology Telescope (NTT) for our observations. The SoFI instrument is equipped with a HAWAII $\mathrm{HgCdTe}$ detector of $1024 \times 1024$ pixels, characterized by a $5.4 \mathrm{e}^{-} / \mathrm{ADU}$ gain, a readout noise of 2.1 ADU, and a dark current of less than $0.1 \mathrm{e}^{-} \mathrm{s}^{-1}$. We observed in the large field mode, giving a $4.9 \times$ $4.9 \operatorname{arcmin}^{2}$ field. All measurements were made through the $J$, $H$, and $K_{\mathrm{S}}$-band filters.

Using a backup program, additional observations of some OGLE planetary transit candidates (taken from those previously observed in the near-IR bands) were performed with the SMARTS $1.0 \mathrm{~m}$ telescope at the Cerro Tololo International Observatory (CTIO) in the $V$ and $I$ optical bands. In this sub sample we included one target previously observed by Gallardo et al. (2005) i.e., OGLE-TR-111 (a confirmed planet), and 5 eclipsing binary systems observed and analyzed by Pont et al. (2005) i.e., OGLE-TR-72, OGLE-TR-78, OGLE-TR-105, OGLE-TR-106 and OGLE-TR-123, to compare their results with our conclusions (see discussion in Sect. 5). The camera was the Y4KCam with STA $4064 \times 4064$ CCD, 15 -micron pixels mounted in an $\mathrm{LN}_{2}$ dewar, and a field of view $20 \times 20 \mathrm{arcmin}^{2}$ square, providing a scale of 0.289 -arcsec/pixel. The data were obtained during two consecutive nights on 2009 March 14, 15. The fields were monitored in both the $V$ and $I$ band filters with 300 and $180 \mathrm{~s}$ exposure times for each image, respectively.

\section{Data reduction}

\subsection{Near-IR photometry}

All data reduction was performed using IRAF tasks ${ }^{1}$. First, a cross-talk correction was applied, taking into account the difference in detectors sensitivity between the upper and the lower half. The sky substraction was then applied. The whole data set was acquired using "dither" mode (regular offset for a specific number of images). These contiguous sky images were used to generate local sky background images close in time to each of the offset images. The appropriately scaled skies were then

\footnotetext{
1 IRAF is distributed by the NOAO, operated by Universities for Research in Astronomy, Inc.
}

subtracted from the images. Finally, we applied flat-field corrections to and aligned all the images. To produce the flat-fiels, we used the correction images provided by the NTT SciOps team ${ }^{2}$, and the alignment was performed with both the lintran and imshift task.

The calibration of the near-IR photometry was performed using 2MASS, by considering common stars between our fields and stars listed in the 2MASS Point Source Catalog. In this calibration, we obtained a zeropoint of $0.1 \mathrm{mag}$ for the $K_{\mathrm{S}}$-band photometry. Some stars used for the calibrations were checked against the Deep Near-Infrared Survey (DENIS) sources.

The average errors for the candidates are smaller than 0.1 mag for the adopted near-IR filters. Finally, some candidates were not included in the analysis because their data was of too low quality.

\subsection{Optical photometry}

The reduction of the $V$ and $I$ band data was performed using the IRAF phot task. We selected an aperture size of 6 pixels and an annulus of 2 pixels to substract the sky background. The transformation to the standard system was performed on the basis of the observations of three Landoldt's (1992) fields using the Bouger linear relation by fitting the instrumental magnitudes for different airmass observations. The target stars have magnitude ranges of $14.50 \leq V \leq 18.69 \mathrm{mag}$ and $13.79 \leq I \leq 16.72 \mathrm{mag}$ with errors that should not exceed 0.05 and 0.07 mag, respectively.

\section{Determining the stellar parameters}

This is the second paper using the same analysis presented in Gallardo et al. (2005), thus, we briefly discuss the main equations developed to determine physical parameters of the observed objects. Two steps are necessary to characterize each star. Using the limb-darkened angular diameter of the star $\left(\theta_{\mathrm{LD}}\right)$, we can infer intrinsic magnitudes from the relations

$\log \left(\frac{\theta_{\mathrm{LD}}}{\operatorname{mas}}\right)=0.5+0.1(V-K)_{\mathrm{o}}-0.2 K_{\mathrm{o}}$,

$\log \left(\frac{\theta_{\mathrm{LD}}}{\text { mas }}\right)=0.5+0.2(I-K)_{\mathrm{o}}-0.2 K_{\mathrm{o}}$

where the index "o" refers to unreddened values, rms dispersions are smaller than $1 \%$ and $3.7 \%$, respectively (Kervella et al. 2004), and the final estimate is the weighted mean of these estimates. We can then infer the effective temperature from the relation

$\log T_{\text {eff }}=4.2-\left[1.2 \log \theta_{\mathrm{LD}}+0.2 K_{\mathrm{o}}-0.6\right]^{1 / 2}$.

Finally, a combination of the relations in Eqs. (1)-(3) yields an estimate of $R_{*}$ by its dependence on the assumed distance as

$$
\left(\frac{R_{*}}{R_{\odot}}\right)=4.4 \times 10^{10}\left(\frac{d}{\mathrm{kpc}}\right) \tan \left(\frac{\theta_{\mathrm{LD}}}{2}\right) .
$$

The second step is based on the properties of main-sequence stars, i.e., the strong correlation both between luminosity and mass $\left(L \sim M^{\beta}\right)$, and between mass and radius $\left(M \sim R^{\alpha}\right)$, hence between effective temperature and radius of the form

$$
\left(\frac{T_{\text {eff }}}{T_{\text {eff } \odot}}\right)=\left(\frac{R_{*}}{R_{\odot}}\right)^{(\alpha \beta-2) / 4} \approx\left(\frac{R_{*}}{R_{\odot}}\right)^{0.64},
$$

\footnotetext{
2 See http://www.ls.eso.org/lasilla/sciops/ntt/sofi/ reduction/flat_fielding.html
} 
using the standard values of $\alpha=1 / 0.8, \beta \approx 3.6$, and solar effective temperature $T_{\text {eff } \odot}=5770 \mathrm{~K}$. In this way, if the distance is known, both estimates agree and the parameters are fully consistent with each other. On the other hand, if there is no distance solution, then either the star is a giant (for which Eqs. (1)-(3) do not apply), or the assumed reddening at that distance is incorrect (and it is then necessary to calculate $\left.(I-K)_{\mathrm{o}}\right)$. The companion radius is then finally derived using the standard relation from Seager \& Mallen-Ornelas (2003)

$\left(\frac{R_{\mathrm{c}}}{R_{*}}\right)=\left(10^{\Delta I / 2.5}-1\right)^{1 / 2}$,

where $\Delta I$ is the depth of the transit in magnitudes in the $I$ band, provided by OGLE.

\section{Constraints on the stellar objects and companions}

Table 1 lists the inferred stellar properties. In Col. (1), we provide the name of the target. The derived distance and effective temperature of the host star are given in Cols. (2) and (3), respectively. The calculated companion radius and its error are shown in Cols. (4) and (5), respectively. The stellar radius is indicated in Col. (6) and its corresponding error is given in Col. (7). The transit depth in the $I$ band is displayed in Col. (8). Finally, Col. (9) indicates the observed orbital period.

As this table shows, there are many M-type stars (Col. (3)) in this sample. Analyzing these results, several companions have radii that are comparable to those of the known close-in giant transiting extrasolar planets and, even more interestingly, some planetary companion candidates are orbiting late M-type stars, as Fig. 1 shows. This figure displays the effective temperature of the host star versus the derived transiting companion's radius.

We performed the following selection steps to determine the most promising candidates to host exoplanets. First, we analize both the spectral types and effective temperatures, using Table 1 and Fig. 2, where we plot the $V-I$ and $I$ absolute values for the 27 stars in filled squares, along with the locus of the main-sequence stars, indicated by a solid line. Thus, we selected the following M-type star candidates: OGLE-TR-61, OGLE-TR-74, OGLE-TR-123, OGLE-TR-143, OGLE-TR-161, OGLE-TR-167, OGLE-TR-172, and OGLE-TR-173. Accroding to the measured companion radii, which agree with the median radii of exoplanets found by transits, only the candidates OGLE-TR-61, OGLE-TR-74, OGLE-TR-123, OGLE-TR-167, and OGLE-TR-173 are promising stars to host extrasolar planets, meriting additional spectroscopic observations. Pietrukowicz et al. (2010) conclude that the shape of the transit OGLE-TR-167 excludes its planetary nature, from their analisys based on VIMOS+VLT photometry. Pont et al. (2005) presented a spectroscopic follow-up of OGLE-TR-123, but claimed that more data were required to confirm the planetary nature of the afore mentioned object. Their tentative result indicates that OGLE-TR-123 may be orbited by a brown dwarf or lowmass star close to the hydrogen-burning limit, making OGLETR-123 an extremely interesting candidate. The possible substellar companion to the other four stellar objects (i.e., OGLETR-61, OGLE-TR-74, OGLE-TR-123, and OGLE-TR-173) orbit M-type stars. These are very interesting in their own right, because they appear to have small-size stellar companions such as HD-149026b (Sato et al. 2005), HAT-P-3b (Torres et al. 2007), or OGLE-TR-111b (Minniti et al. 2007), with radius ranging from $\sim 0.5 R_{\text {Jup }}$ to $1.0 R_{\text {Jup }}$. We have also demostrated

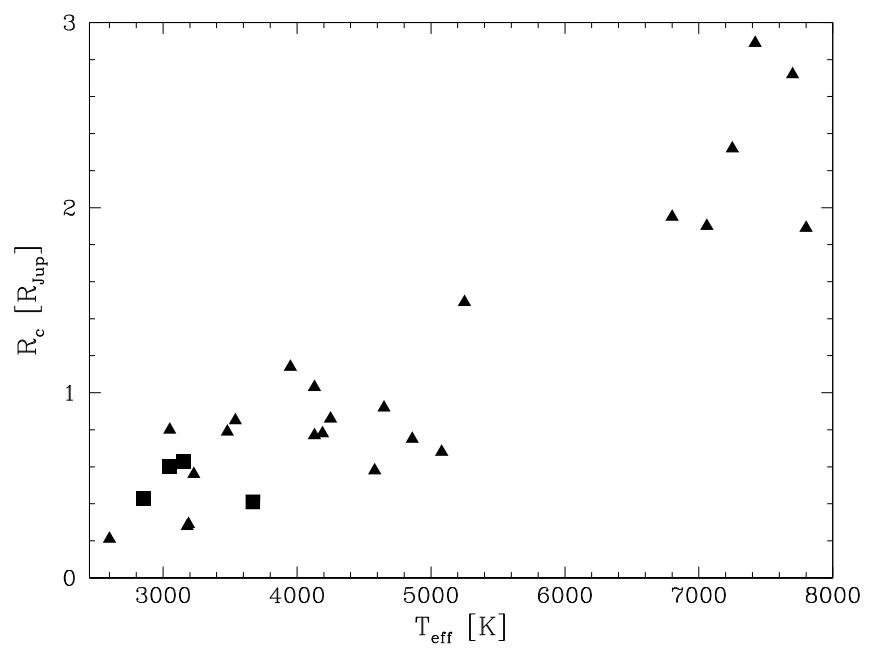

Fig. 1. Radius for the companions versus effective temperature of the host star for 27 observed OGLE objects. Error in $T_{\text {eff }}$ is of $\pm 600[\mathrm{~K}]$. Filled squares indicate the promising candidates around M-type stars while the remainder of the sample is indicated with filled triangles.

the reliability of the method proposed by Gallardo et al. (2005), by confirming OGLE-TR-111 (Minniti et al. 2007) to be an exoplanet host star that has both an effective temperature and companion radius in total consistency with our estimations.

We note that some K-type stars with well-measured companion radii, should also be considered as good transit candidates. These are OGLE-TR-71, OGLE-TR-72, OGLE-TR-158, and OGLE-TR-164. One of these candidates, OGLE-TR-72, was however discarded in accordance with Pont et al. (2005), who classified it as an M eclipsing binary system.

Finally, using the same method described above, we selected OGLE-TR-60, OGLE-TR-155, OGLE-TR-160, OGLE-TR-162, and OGLE-TR-163 as additional good transit candidates to host exoplanets, these stars probably being F or G spectral type.

On the other hand, we rejected all remaining candidates for the following reasons: (1) we find OGLE-TR-75 to have substantial inconsistencies in terms of magnitude for estimating accurate stellar properties; (2) OGLE-TR-70, OGLE-TR-156, and OGLE-TR-159 have inconsistencies when comparing effective temperature inferred and spectral type classification; (3) either OGLE-TR-78, OGLE-TR-105, OGLE-TR-106, OGLE-TR-142, and OGLE-TR-146 are giants because the method based on both the well-calibrated surface brightness relation and the correlation between mass and luminosity for main-sequence stars, does not provide acceptable solutions, or their distances have been estimated with errors that are far too large. We note that candidates OGLE-TR-78, OGLE-TR-105, and OGLE-TR-106 were classified by Pont et al. (2005) as M, G, and M binary systems, respectively, making our selection criteria result for these candidates a robust conclusion. Finally, candidates OGLE-TR-143, OGLE-TR-161, and OGLE-TR-172 have low-mass companions whose radii are far too small to be good transit candidates following our selection criteria.

\section{Conclusions}

In this second paper we have presented, a continuation of the results presented in Gallardo et al. (2005), by analyzing optical and near-IR photometry of candidate OGLE transits. We have 
Table 1. Physical properties for the 27 observed OGLE objects and their companions.

\begin{tabular}{ccccccccc}
\hline \hline $\begin{array}{c}\text { Name } \\
\text { OGLE-TR- }\end{array}$ & $\begin{array}{c}d^{a} \\
{[\mathrm{kpc}]}\end{array}$ & $\begin{array}{c}T_{\text {eff }}^{b} \\
{[\mathrm{~K}]}\end{array}$ & $\begin{array}{c}R_{\mathrm{c}}{ }^{c} \\
{\left[R_{\text {Jup }}\right]}\end{array}$ & $\begin{array}{c} \pm R_{\mathrm{c}} \\
{\left[R_{\text {Jup }}\right]}\end{array}$ & $\begin{array}{c}R_{*} \\
{\left[R_{\odot}\right]}\end{array}$ & $\begin{array}{c} \pm R_{*} \\
{\left[R_{\odot}\right]}\end{array}$ & $\begin{array}{c}\Delta I \\
{[\mathrm{mag}]}\end{array}$ & $\begin{array}{c}P \\
{[\text { days }]}\end{array}$ \\
\hline 60 & 2.35 & 7800 & 1.89 & 0.30 & 1.60 & 0.26 & 0.016 & 2.31 \\
61 & 0.25 & 3050 & 0.60 & 0.06 & 0.37 & 0.04 & 0.030 & 4.26 \\
70 & 0.25 & 3050 & 0.80 & 0.08 & 0.36 & 0.04 & 0.053 & 8.04 \\
71 & 0.80 & 4250 & 0.86 & 0.06 & 0.62 & 0.05 & 0.022 & 4.19 \\
72 & 0.65 & 3950 & 1.14 & 0.09 & 0.55 & 0.04 & 0.048 & 6.85 \\
74 & 0.25 & 3150 & 0.63 & 0.06 & 0.39 & 0.04 & 0.030 & 1.58 \\
75 & 1.55 & 5250 & 1.49 & 0.22 & 0.86 & 0.13 & 0.034 & 2.64 \\
78 & 2.40 & 7250 & 2.32 & 0.40 & 1.43 & 0.25 & 0.03 & 5.32 \\
105 & 2.80 & 6800 & 1.95 & 0.54 & 1.29 & 0.36 & 0.026 & 3.06 \\
$106^{*}$ & 6.00 & 7060 & 1.90 & 0.10 & - & - & 0.022 & 2.54 \\
$111^{*}$ & 0.80 & 4650 & 0.92 & 0.06 & 0.71 & 0.05 & 0.019 & 4.02 \\
123 & 0.50 & 3670 & 0.41 & 0.03 & 0.49 & 0.04 & 0.008 & 1.80 \\
$142^{*}$ & 3.80 & 7700 & 2.72 & 0.1 & - & - & 0.034 & 3.06 \\
143 & 0.04 & 2600 & 0.21 & 0.02 & 0.29 & 0.03 & 0.006 & 3.34 \\
$146^{*}$ & 5.80 & 7420 & 2.89 & 0.1 & - & - & 0.043 & 2.94 \\
155 & 1.10 & 5080 & 0.68 & 0.06 & 0.82 & 0.08 & 0.008 & 5.27 \\
156 & 0.25 & 3480 & 0.79 & 0.07 & 0.45 & 0.04 & 0.034 & 3.58 \\
158 & 0.70 & 4130 & 0.77 & 0.06 & 0.59 & 0.04 & 0.019 & 6.38 \\
159 & 0.45 & 3540 & 0.85 & 0.08 & 0.47 & 0.04 & 0.038 & 2.12 \\
160 & 0.50 & 4580 & 0.58 & 0.04 & 0.70 & 0.05 & 0.008 & 4.90 \\
161 & 0.25 & 3180 & 0.28 & 0.03 & 0.39 & 0.04 & 0.006 & 2.74 \\
162 & 0.75 & 4860 & 0.75 & 0.05 & 0.76 & 0.05 & 0.011 & 3.75 \\
163 & 0.65 & 4130 & 1.03 & 0.08 & 0.59 & 0.04 & 0.034 & 0.94 \\
164 & 0.75 & 4190 & 0.78 & 0.06 & 0.61 & 0.05 & 0.019 & 2.68 \\
167 & 0.25 & 3230 & 0.56 & 0.05 & 0.40 & 0.04 & 0.022 & 5.26 \\
172 & 0.20 & 3190 & 0.29 & 0.03 & 0.40 & 0.04 & 0.006 & 1.79 \\
173 & 0.10 & 2850 & 0.43 & 0.05 & 0.33 & 0.04 & 0.019 & 2.60 \\
\hline & & & & & & & &
\end{tabular}

Notes. ${ }^{(a)}$ Errors in the distance correspond to $\pm 0.25 \mathrm{kpc}$ on average. ${ }^{(b)}$ Errors in the effective temperature correspond to $\pm 600 \mathrm{~K} .{ }^{(c)} R_{\mathrm{Jup}}=0.103$ $R_{\odot} \cdot{ }^{(*)}$ Presumable giant stars (see discussion in Sect. 5). ${ }^{(*)}$ Already confirmed planet.

Table 2. Physical properties for the 27 observed OGLE objects and their companions.

\begin{tabular}{cccrrr}
\hline \hline $\begin{array}{c}\text { Name } \\
\text { OGLE-TR- }\end{array}$ & $\begin{array}{c}V \\
{[\mathrm{mag}]}\end{array}$ & $\begin{array}{c}I \\
{[\mathrm{mag}]}\end{array}$ & $\begin{array}{c}J \\
{[\mathrm{mag}]}\end{array}$ & $\begin{array}{c}H \\
{[\mathrm{mag}]}\end{array}$ & $\begin{array}{c}K \\
{[\mathrm{mag}]}\end{array}$ \\
\hline 60 & 15.25 & 14.59 & 14.16 & 14.07 & 13.81 \\
61 & 18.17 & 16.33 & 14.82 & 14.27 & 13.87 \\
70 & 17.87 & 16.68 & 15.7 & 14.18 & 13.93 \\
71 & 17.55 & 16.22 & 15.61 & 15.13 & 14.54 \\
72 & 17.78 & 16.41 & 15.61 & 15.13 & 14.54 \\
74 & 17.74 & 15.99 & 14.37 & 13.95 & 13.58 \\
75 & 16.18 & 16.86 & 15.96 & 14.87 & 14.84 \\
78 & 16.24 & 15.71 & 14.93 & 14.68 & 14.64 \\
105 & 16.93 & 15.93 & 15.09 & 14.72 & 14.9 \\
106 & 17.84 & 16.72 & 15.99 & 16.04 & 16.3 \\
111 & 16.93 & 15.6 & 14.7 & 14.22 & 14.11 \\
123 & 18.69 & 15.81 & 14.64 & 14.31 & 14.21 \\
142 & 16.22 & 15.32 & 15.18 & 14.86 & 14.9 \\
143 & 16.31 & 13.79 & 12.1 & 11.1 & 10.73 \\
146 & 17.79 & 16.43 & 15.73 & 15.41 & 16.02 \\
155 & 16.77 & 15.68 & 14.82 & 14.44 & 14.29 \\
156 & 16.39 & 15.31 & 13.78 & 13.15 & 12.98 \\
158 & 17.56 & 16.01 & 14.89 & 14.3 & 14.29 \\
159 & 17.84 & 16.43 & 15.32 & 14.04 & 14.17 \\
160 & 15.53 & 14.44 & 13.57 & 13.18 & 13 \\
161 & 17.59 & 15.80 & 14.5 & 13.76 & 13.55 \\
162 & 16.17 & 15.00 & 14.09 & 13.81 & 13.67 \\
163 & 17.16 & 15.90 & 14.99 & 14.4 & 14.14 \\
164 & 17.66 & 16.23 & 15.07 & 14.54 & 14.51 \\
167 & 17.85 & 15.71 & 14.41 & 13.68 & 13.41 \\
172 & 17.49 & 15.41 & 13.93 & 13.18 & 12.99 \\
173 & 16.96 & 14.93 & 13.31 & 12.52 & 12.34 \\
\hline & & & & &
\end{tabular}

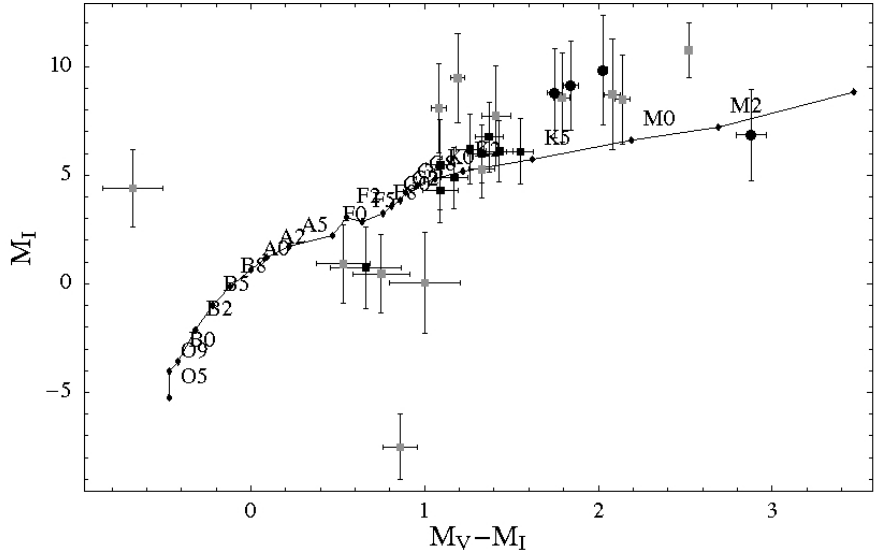

Fig. 2. Absolute color-magnitude diagram. Filled black circles denote the most promising M-type candidates. The filled black squares denote promising candidates around F- and/or G-type stars. On the other hand, discarded candidates are represented by grey filled squares. Error bars correspond mainly to distance uncertainties. The main-sequence stars loci are plotted as a solid black line.

performed a self-consistent characterization of systems identified by the OGLE collaboration. By combining near-IR and optical observations, we have derived stellar parameters such as effective temperature, distance, mass, and radii of 27 transiting candidates using the method presented in Gallardo et al. (2005). This method has provided the accurate determination of the aforementioned astrophysical properties, allowing us to refine 
our estimates of the radii of the companions and perform an efficient selection of possible extrasolar planets.

Our main conclusions are:

(1) The objects OGLE-TR-70, OGLE-TR-72, OGLE-TR-75, OGLE-TR-78, OGLE-TR-105, OGLE-TR-106, OGLETR-142, OGLE-TR-143, OGLE-TR-146, OGLE-TR-156, OGLE-TR-159, OGLE-TR-161, OGLE-TR-167, and OGLE-TR-172 can be excluded from future observations.

(2) We select $M$ or K-type star candidates OGLE-TR-61, OGLE-TR-71, OGLE-TR-74, OGLE-TR-123, OGLE-TR158, OGLE-TR-164, and OGLE-TR-173 as very good transit candidates to host exoplanets.

(3) We have also selected OGLE-TR-60, OGLE-TR-155, OGLE-TR-160, OGLE-TR-162, and OGLE-TR-163 as some other possible main-sequence stars harboring planets. Dreizler et al. (2007) selected OGLE-TR-138 (not included in our analysis), OGLE-TR-158, OGLE-TR-160 and OGLE-TR-163 as possible stars harboring planets. We agree in the classification of 3 of their 4 best candidates. Moreover, our estimates of the $T_{\text {eff }}$ and $R_{\mathrm{c}}$ are also in agreement with their work. This confirms the reliability of our present selection. Finally, we note that candidates indicated in conclusions item (2) are all $\mathrm{M}$ or $\mathrm{K}$ spectral type stars that potentially represent the first detections of planetary companions orbiting a stellar object with this spectral type. Only one exoplanet transiting an M-type dwarf is known: Glise 436b (Butler et al. 2004; Gillon et al. 2007). In this way, we mostly select candidates OGLE-TR-61, OGLE-TR-74, OGLE-TR-123, and OGLE-TR-173 as the most promising M-type stars to host extrasolar planets, and plan to obtain high-resolution follow-up observations to confirm our results by determining the masses of the planets accurately, and to provide more insight into their nature.

Acknowledgements. D.M. and P.P. are supported by FONDAP Center for Astrophysics 15010003, BASAL Center for Astrophysics and Associated Technologies PFB-06. D.M. is also supported by MIDEPLAN Milky Way Millennium Nucleus. P.P. was also supported by the Foundation for Polish Science through program MISTRZ and the Polish Ministry of Science and Higher Education through the grant N N203 301335.

\section{References}

Alonso, R., Deeg, H. J., Brown, T. M., \& Belmonte, J. A. 2004, ESASP, 538, 255

Butler, R. P., Vogt, S. S., Marcy, G. W., et al. 2004, ApJ, 617, 580

Charbonneau, D., Brown, T., Latham, D. W., \& Mayor, M. 2000, ApJ, 529, L45 Drake, A. 2003, ApJ, 589, 1020

Dreizler, S., Rauch, T., Hauschildt, P., et al. 2002, A\&A, 319, L17

Dreizler, S., Schuh, S., \& Homeier, D. 2007, A\&A, 464, 367

Gallardo, J., Minniti, D., Valls-Gabaud, D., \& Rekjuba, M. 2005, A\&A, 431, 707

Gillon, M., Pont, F., Demory, B.-O., et al. 2007, A\&A, 472, L13

Henry, G. W., Marcy, G. W., Butler, R. P., \& Vogt, S. S. 2000, ApJ, 529, L41

Kervella, P., Thévenin, F., Di Folco, E., \& Ségrasan, D. 2004, A\&A, 426, 297

Léger, A., Rouan, D., Schneider, J., et al. 2009, A\&A, 506, 287

Minniti, D., Fernández, J. M., Díaz, R. F., et al. 2007, ApJ, 660, 858

Pietrukowicz, P., Minniti, D., Diaz, R. F., et al. 2010, A\&A, 509, A4

Pont, F., Bouchy, F., Melo, C., et al. 2005, A\&A, 438, 1123

Sato, B., Fisher, D., Henry, G. W, et al. 2005, ApJ, 633, 465

Seager, S., \& Mallén-Ornelas, G. 2003, ApJ, 585, 1038

Silva, A. V. R., \& Cruz, P. C. 2006, ApJ, 642, 488

Sirko, E., \& Paczyński, B. 2003, ApJ, 592, 1217

Torres, G., Bakos, G. Á., Kovács, G., et al. 2007, ApJ, 666, L121

Udalski, A., Paczynski, B., Zebrun, K., et al. 2002, Acta Astron., 52, 317

Udalski, A., Pietrzynski, G., Szymanski, M., et al. 2003, Acta Astron., 53, 133

Udalski, A., Szymanski, M. K., Kubiak, M., et al. 2004, Acta Astron., 54, 313 